\title{
Step Characterization using Sensor Information Fusion and Machine Learning
}

\author{
${ }^{1}$ Ricardo Anacleto, ${ }^{23}$ Lino Figueiredo, ${ }^{3}$ Ana Almeida, ${ }^{14}$ Paulo Novais and ${ }^{3}$ António Meireles \\ ${ }^{1}$ ALGORITMI research group at University of Minho, Portugal \\ ${ }^{2}$ Electrical Engineering Department, Institute of Engineering of Porto, Portugal \\ ${ }^{3}$ Knowledge Engineering and Decision Support (GECAD) University of Minho, Portugal \\ ${ }^{4}$ Computer Science at the Department of Informatics, in the School of Engineering of the University of Minho, Portugal
}

\begin{abstract}
A pedestrian inertial navigation system is typically used to suppress the Global Navigation Satellite System limitation to track persons in indoor or in dense environments. However, lowcost inertial systems provide huge location estimation errors due to sensors and pedestrian dead reckoning inherent characteristics. To suppress some of these errors we propose a system that uses two inertial measurement units spread in person's body, which measurements are aggregated using learning algorithms that learn the gait behaviors. In this work we present our results on using different machine learning algorithms which are used to characterize the step according to its direction and length. This characterization is then used to adapt the navigation algorithm according to the performed classifications.
\end{abstract}

Keywords-Pedestrian Inertial Navigation System, Indoor Location, Learning Algorithms, Information Fusion

\section{INTRODUCTION}

LOCATION information is an important source of context for ubiquitous systems, as it can be explored to improve life quality since emergency teams [1] can respond more precisely if the team members location is known, tourists can have better recommendations [2], the elderly can be better monitored [3], parents can be more relaxed with their children in shopping malls [4] and presence control systems can produce better resits [22].

The major limitation of these systems is related to retrieving individual's location, which nowadays is only based on a GNSS (Global Navigation Satellite System), restricting the use of these systems to environments where GNSS signals are available. However, GNSS signals are not available inside buildings, in urban canyons, in the underground, underwater and in dense forests. Consequently location-aware applications sometimes cannot know the user location. Therefore, developing complementary localization technologies for these environments would unleash the use of many applications as presented above [23].

There are already some proposed systems that retrieve location in indoor environments. However, most of these solutions require a structured environment [5]. Therefore, these systems could be a possible solution for indoor environments, but in a dense forest or in urban canyons they are very difficult to implement.

To suppress structured environment limitations, a Pedestrian Inertial Navigation Systems (PINS) can be used. Typically, a PINS is based on an algorithm that involves three phases: step detection, step length estimation and heading estimation. A PINS uses accelerometers, gyroscopes, among other sensors, to continuously calculate via dead reckoning the position and orientation of a pedestrian. These sensors are based on MEMS (Microelectromechanical systems), which are tiny and lightweight sensors, making them ideal to integrate into the person's body. Unfortunately, large deviations of inertial sensors can affect performance, so the PINS big challenge is to correct the sensors deviations.

In the previous works of the research team, the step detection was improved by using an algorithm that combines an accelerometer and force sensors placed on the pedestrian's foot [6]. This approach led to better results [7] on the estimation of the pedestrian displacement. However, it still exists an error of $0.4 \%$ in step detection and an error of $7.3 \%$ in distance estimation.

We have found that a PINS solution only based on one IMU (Inertial Measurement Unit), composed by an accelerometer and a gyroscope, is not accurate enough. Thus, we believe that using several IMU in the person's body, combined with an information fusion strategy, will improve the accuracy of a PINS.

Information fusion is a multi-disciplinary research field with a wide range of potential applications in areas such as defense, robotics, automation and pattern recognition. During the past two decades, extensive research and development on multiple sensor data fusion has been performed for the Department of Defense of the United States of America [8]. This subject has been and will continue to be an everincreasing interest field in research community, where it is intended to develop more advanced information fusion methodologies and architectures.

In the case of PINS, the MEMS sensors have some limitations and low accuracy, which does not happen on more expensive sensors like the ones used on aviation and military applications. To reduce the sensors complexity and thereby its cost, the information from a set of simple and low-cost sensors can be combined. This leads to the creation of a less expensive system, which captures accurate and reliable information about the pedestrian movements. Moreover, this fusion turns the system more fault tolerant [21].

Information fusion combined with learning techniques are being used in different INS fields to assist in displacement estimation. In robotics, Faceli et al. [9] use these techniques to improve the accuracy of distance measurements between a robot and the objects present in the environment by $7 \%$.

These techniques are also used in autonomous driving vehicles. Stanley [10] software relied on machine learning and probabilistic reasoning techniques. Its IMU combined with artificial intelligence techniques were able to maintain accurate pose of the vehicle during GPS outages of up to 2 minutes.

In land vehicle applications, Caron et al. [11] and Noureldin et al. [12] propose machine learning techniques like neural networks, which introduce context variables and errors modelling for each sensor. Authors conclude that with an adequate modelling an accuracy 


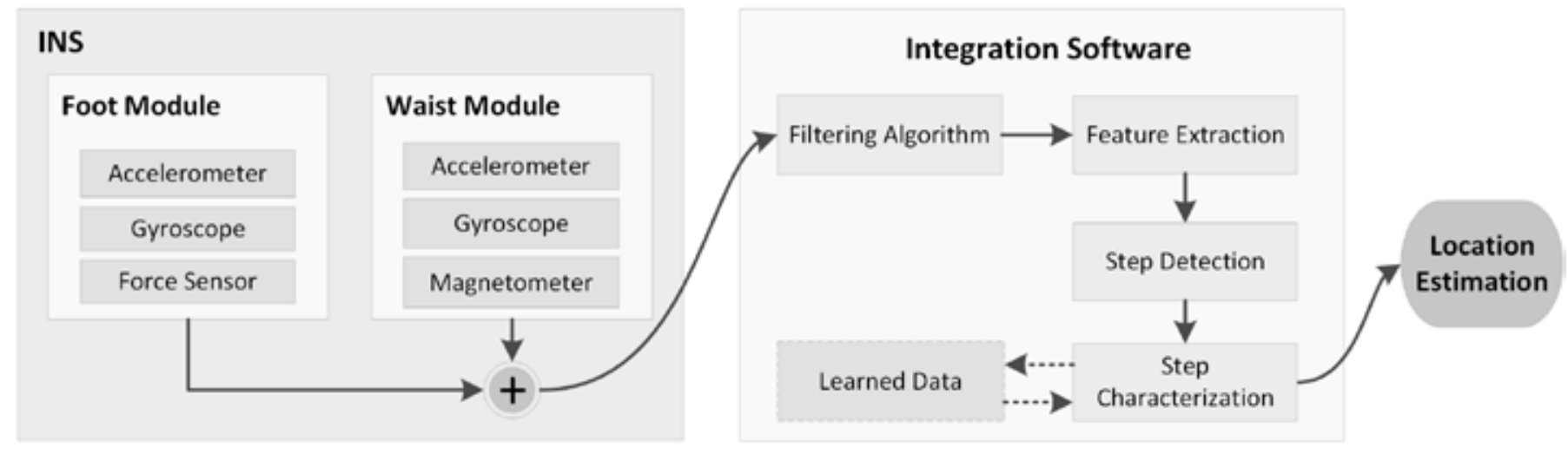

Figure 1 - System architecture.

improvement of $20 \%$ can be achieved. Recently, Noureldin et al. [13] have improved the previous results by considering past samples of INS position and velocity errors. Bhatt et al. [14] propose a hybrid data fusion methodology using Dempster-Shafer theory augmented by a trained Support Vector Machine (SVM), which corrects the INS errors. The proposed methodology has shown an accuracy improvement of $20 \%$.

Since these experiences presented good results in the respective area, we wanted to explore similar techniques but applied to PINS. Our proposal applies an information fusion from several IMU spread in the person's body, and learning algorithms that based on contextual and past examples can improve the PINS accuracy. However, we needed to understand which is the best machine learning model to be used in a PINS.

This goal is addressed throughout the document, where the system architecture is presented in Section II. In the following three sections, Section III, IV and V, are presented the machine learning algorithms that were used to characterize a step. This characterization is applied to limit the typical error growing of PINS. In these sections is presented a comparison between a SVM and a Neural Network. In Section III are presented the algorithms that characterize the step according to the type of terrain, normal (flat) or stairs (i.e. ascending or descending).

In Section IV are presented the algorithms that characterize the step as forward or backward, and in Section V are presented the algorithms that classify a step according to its size (i.e. short, normal and long). In each section is presented an evaluation made to each algorithm. Finally, in Section VI are discussed the conclusions and the future work.

\section{System Architecture}

The proposed system is composed by two low-cost IMU, developed by the authors [6], and an "Integration Software" (described in Sections III, IV and V). The "Integration Software" starts by filtering the signals obtained from the sensors, then some features are extracted, which are used to detect a step and thereby to characterize it according to some previously learned data. Finally, the displacement is estimated based on the collected information. This architecture is represented in Figure 1.

When referring to a low-cost IMU it implies different things for researchers, since for some a thousand euros IMU is considered low-cost. However, in a PINS a low-cost IMU should cost less than $100 €$. This price restriction, implies the use of MEMS sensors that are truly low-cost.

The first IMU (Waist IMU), represented in Figure 2, is placed on the abdominal area and is composed by a STMicroelectronics L3G4200D gyroscope [15], a Analog Devices ADXL345 accelerometer [16] and a Honeywell HMC5883L magnetometer [17].

The second IMU (Foot IMU) is placed on the foot and is represented in Figure 3. It is composed by an Analog Devices ADXL345 accelerometer [16], a STMicroelectronics L3G4200D gyroscope [15] and two Tekscan FlexiForcer A201 force sensors [18]. Typically, an accelerometer is used to detect and quantify the foot movement, and the gyroscope is valuable to transform this acceleration data from the sensor frame to the navigation frame.

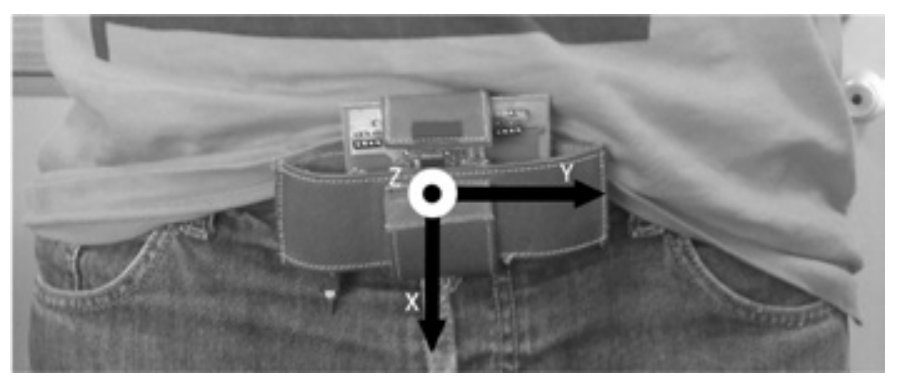

Figure 2 - Waist BSU with the corresponding axis

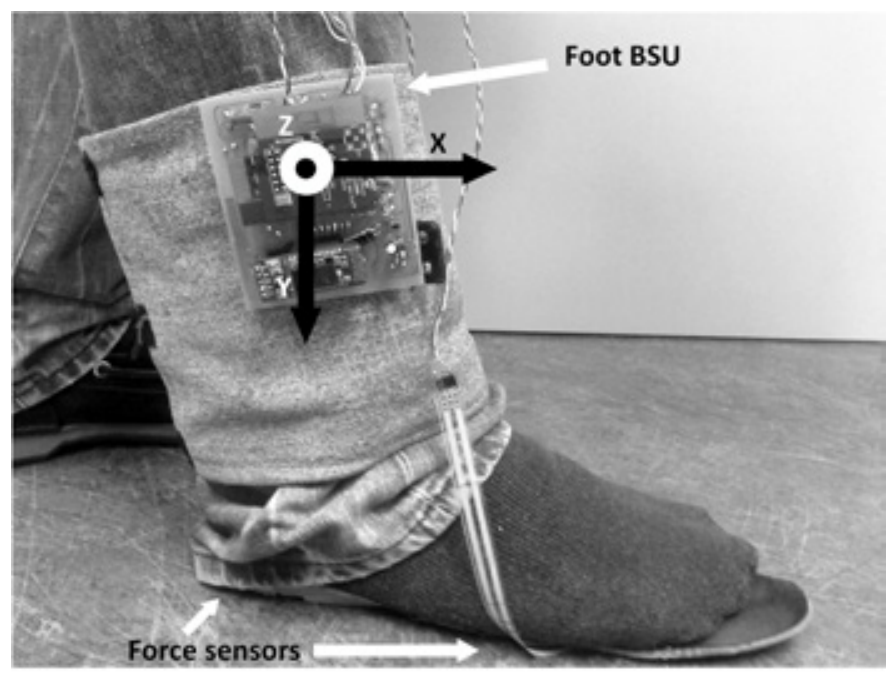

Figure 3 - Foot BSU with the corresponding axis

Force sensors were included since they can improve the detection of the moments when the user touches his feet on the ground, as well as, the correspondent contact force. The combination of force sensor data with accelerometer data improves the accuracy of the step length estimation [7]. One force sensor was placed on the front part of the foot and the other on the heel, as shown in Figure 3.

Although the pattern of the acceleration can be used to classify a step, sometimes the accelerometer produce a signal that does not follow any pattern, which turns to be useless to correctly classify a step.

These random readings can be surpass by using several sources of data combined with learning algorithms. The probability that two 
sources of data give erroneous acceleration patterns at the same step is much reduced. The fusion between all the sensors information can improve the number of correct classifications.

\section{STEP TERRAIN}

The first step characterization that is performed, is about the type of terrain where it was given. There are three possibilities: (i) in a normal (flat) terrain; (ii) or in ascending; (iii) or descending stairs.

For this characterization it was used the data from three sensors: (i) foot accelerometer (y-axis); (ii) foot gyroscope (z-axis); (iii) and waist accelerometer (x-axis).

The $y$-axis of the foot accelerometer provides relevant information about the foot elevation, which is essential to distinguish between ascending or descending stairs, since the forces are the opposite. However, from the several tests performed it was noticed that the main distinction that can be made using this sensor data is between ascending stairs and the other types of terrain. When ascending a stair the foot has to perform a higher elevation than in the other two cases. Regarding the other two types of terrain, descending stairs and normal, the data obtained from this sensor is very similar. The main difference is at the end of the step that, in the case of descending stairs, a higher acceleration is sensed since the foot touches the ground with a higher force than in the normal terrain type.

The $\mathrm{z}$-axis of the foot gyroscope provides information about the foot rotation in each type of terrain. The foot rotation is much more noticeable in the ascending and descending stairs terrains. When ascending stairs it has an upward rotation peak and then a downward rotation peak, and it is the opposite when descending stairs. The data from this sensor is very important to make the distinction between these two types of terrains. Regarding the normal terrain, the pattern is similar to the descending stairs. However, the sensed rotation is much softer. Nonetheless this sensor provides a good accuracy on making the distinction between the three types of terrain.

Finally, the $\mathrm{x}$-axis of the waist accelerometer provides similar data as the foot accelerometer. In ascending stairs a higher acceleration is sensed, in both positive and negative scales. When descending stairs this acceleration is much lower than in the other two types of terrain. The acceleration sensed in the normal terrain is within the other two. It provides similar data to distinguish between a flat surface and descending stairs. However, when ascending stairs it provides distinguishable data.

Considering the data provided by these signals, it can be established that, combining their data, they are suitable to be used to differentiate each possible characterization terrain. Since the strengths of each signal can be combined to achieve a final consensus.

To perform this characterization the learning algorithms were fed with a total of 72 inputs ( 24 inputs per each sensor). Each sensor signal was divided into 6 equal parts, and for each one of these parts the maximum, minimum and mean values were obtained, as well as, the slope. The slope was calculated based on the first and on the last measurement of each part. This data gives a total of 24 inputs per each sensor that are fed into the learning algorithm.

The division of the signal was made because giving to a classifier a complete signal can be very heavy and confusing to the algorithm to identify the patterns of the signal and therefore estimate the correct label for that pattern. Thus, it is reduced the dimensionality of the problem domain for the purposes of improving the performance of the algorithms and to decrease the computational load.

It was decided to divide the signal in 6 parts, because, during a step, each sensor signal is typically composed by 30 measurements. Thus, in order to have an average of 5 measurements per iteration the signal was divided into 6 equal parts. More parts will divide the signal too much, and fewer parts will pass insufficient information to the learning algorithm. Thus, the 6 was the number of parts that have best represented each one of the signals.

The learning algorithms were trained with a total of 970 samples, 358 samples of ascending stairs steps, 358 samples of descending stairs steps and 254 samples of normal terrain steps. To validate the algorithms a total of 170 samples were used (62 ascending, 62 descending and 46 normal). To test the algorithms a total of 540 samples were used (180 ascending, 260 descending and 100 normal), and a 10-fold cross-validation using these datasets was also performed.

\section{A. SVM}

Since in this characterization there are three possible classes (i.e. normal, ascending or descending stairs), and the SVM models can only classify two at each time, three SVM models (SVM Model 1, SVM Model 2 and SVM Model 3) were created. From the executed tests it was identified that the best results were achieved using a kernel, configured as a 3 th order polynomial. This architecture is represented in Figure 4.

The models were trained with the same data, but with different class labels vectors. In this case there are three vectors. The first vector, which is used by the SVM Model 1, indicates that the ascending stairs steps belong to the positive class and the others to the negative. The second vector, which is used by the SVM Model 2, indicates that the descending stairs steps are the positive entries and the other the negatives. The third vector, which is used by the SVM Model 3, indicates that the normal terrain steps are the positive classifications and the others the negative. Meaning that the positive class of each classifier is ascending stairs, descending stairs and normal terrain, respectively.

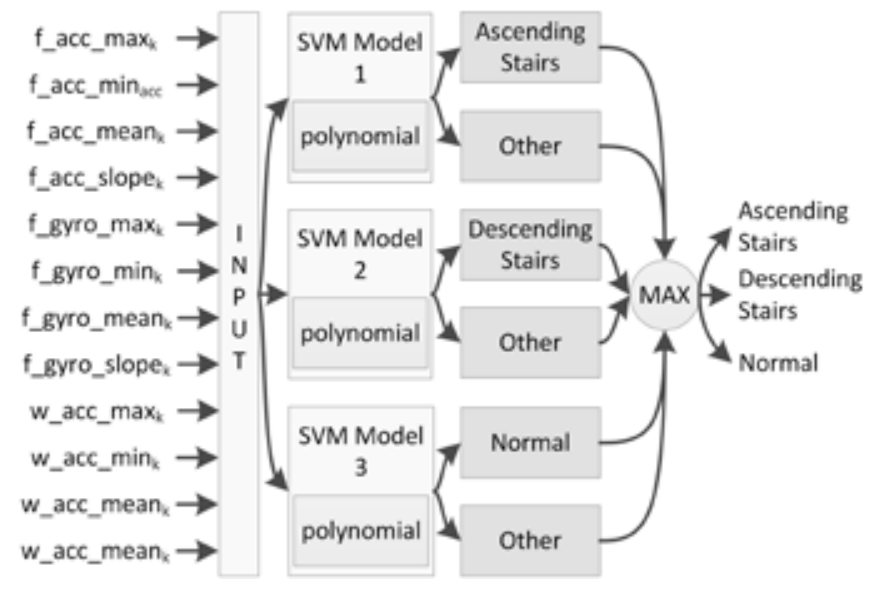

Figure 4 - SVM architecture for step terrain characterization

The score of the new observations are then estimated using each classifier. This will create a vector with three scores, one per each classifier. The index of the element with the highest score is the index of the class to which the new observation most likely belongs. For example, if the first index has the highest value, then the step is classified as ascending stairs. Thus each new observation is associated with the classifier that gives to it the maximum score.

After the learning phase, a 10-fold cross validation to the model was performed. The SVM Model 1 presented no error, the SVM Model 2 presented an error of $0.8 \%$ and the SVM Model 3 presented an error of $2.6 \%$. 


\section{B. Neural Network}

In Figure 5 is represented the design of the implemented neural network that classifies the type of terrain. The neural network receives as input ( $\mathrm{j}$ ) the 72 features previously presented. This input is passed to the Hidden Layer, which is composed by 144 neurons. Then, the Output Layer returns the final result about the type of terrain where the step was given.

The neural network parameters namely, the number of neurons in the hidden layer, the learning rate and the number of iterations, were tuned by trial and error. The learning rate was defined as 0.01 and the number of iterations as 36 .

The mean squared error of the best validation performance was 7.97 $\times 10^{-7}$ with a gradient of $9.80 \times 10^{-7}$ at epoch 36 .

The error given by the neural network is very low, where during the training phase more than $98 \%$ of the results are very close to zero error. The highest error for an instance was of $1.50 \times 10^{-5}$.

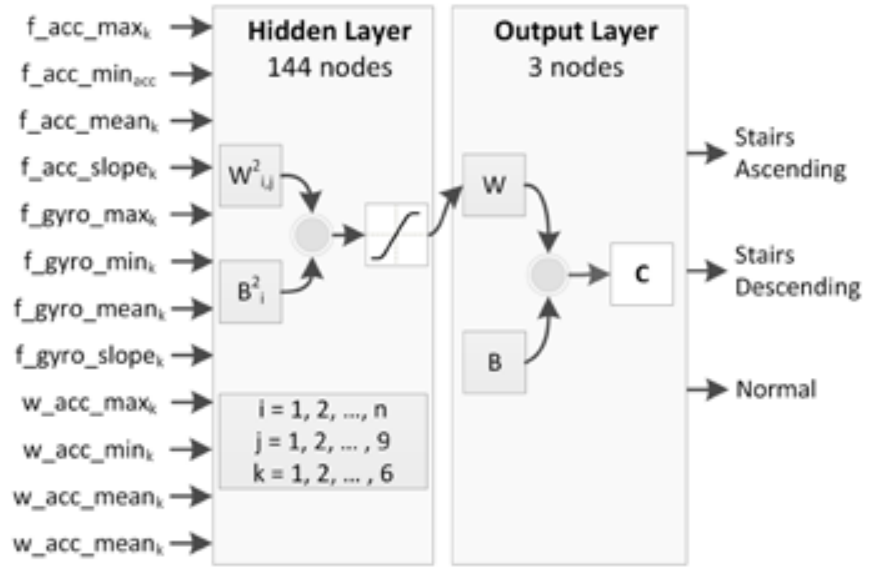

Figure 5 - Neural Network architecture for step terrain characterization

\section{Evaluation}

The implemented algorithms that characterize the type of terrain, were evaluated using a dataset of 800 steps performed by two pedestrians (400 steps for each pedestrian).

The test scenario is represented in Figure 6, which involves a complex path with a set of straight walks and a set of stairs.

The results obtained for this scenario can be seen in Table 1. This table presents for each algorithm, the categorization accuracy (in percentage).

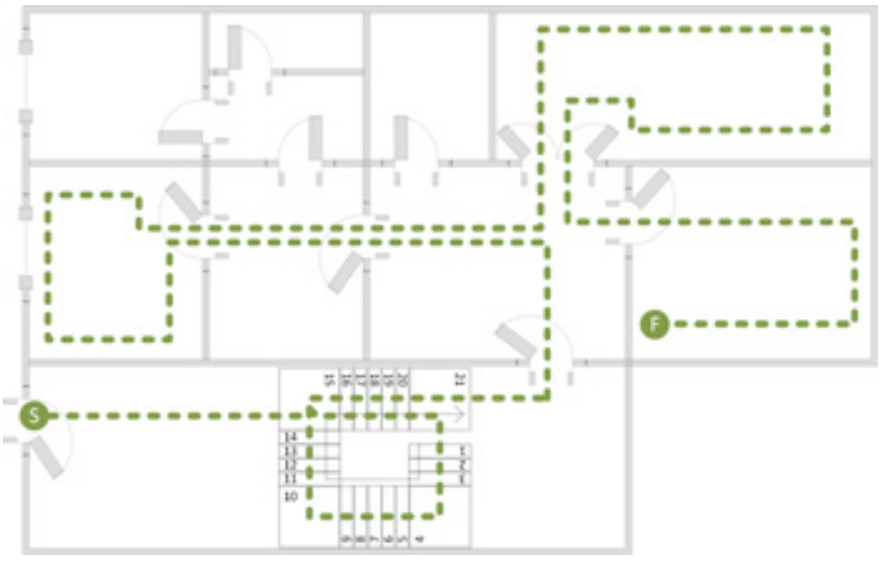

Figure 6 - Evaluation Scenario
For all the algorithms are presented the results obtained, in separate for each BSU and for the combination of the data of both BSU. This allows to identify which one of the BSU has higher accuracy in each characterization type.

TABLE 1 - ACCURACY RESULTS FOR STEP TERRAIN CHARACTERIZATION

\begin{tabular}{ccccccc}
\hline \multirow{2}{*}{ Method } & \multicolumn{2}{c}{ Ascending } & \multicolumn{2}{c}{ Descending } & \multicolumn{2}{c}{ Normal } \\
\cline { 2 - 7 } & Waist & Foot & Waist & Foot & Waist & Foot \\
& BSU & BSU & BSU & BSU & BSU & BSU \\
\hline SVM & $97.5 \%$ & $99.4 \%$ & $94.2 \%$ & $99.4 \%$ & $85.1 \%$ & $94.9 \%$ \\
\hline N.N. & $98.3 \%$ & $100 \%$ & $94.1 \%$ & $100 \%$ & $87.2 \%$ & $94.9 \%$ \\
\hline SVM Fusion & \multicolumn{2}{c}{$99.4 \%$} & \multicolumn{2}{c}{$99.5 \%$} & $96.2 \%$ \\
\hline N.N. Fusion & \multicolumn{2}{c}{$100 \%$} & \multicolumn{2}{c}{$100 \%$} & \multicolumn{2}{c}{$98.7 \%$} \\
\hline
\end{tabular}

Considering the obtained results it can be concluded that the ascending stairs class is the easiest to classify. The normal terrain class is sometimes confused with the descending stairs class, so it is with this misclassification that most errors occur.

Regarding the BSUs, the foot BSU gives more accurate data, since the foot is closer to the ground. The waist BSU can give a good indication about the vertical movement of the body. However, it obtains similar data when descending stairs and in normal terrain. Thus, it presents worst results in these classifications.

Interpreting the results obtained for each algorithm using each BSU in separate, it can be concluded that the Neural Network achieves better results on both BSU locations.

Analyzing the results obtained for each algorithm when considering the fusion of both BSU, the Neural Network presented the best results, achieving a mean accuracy of $99.4 \%$, having $100 \%$ of accuracy on predicting the ascending and descending stairs classes.

Also, it can be concluded that through the sensors complementarity the type of terrain was categorized with higher accuracy.

From our tests it was identified that a learned dataset 5 times smaller, than the used one, is sufficient to achieve similar results. Making the learning procedure simpler and faster to a pedestrian perform before using our system.

Concluding, the evaluation results show that both BSU give similar results on detecting each type of terrain, but with their integration better results can be achieved.

\section{STEP Direction}

The second characterization performed to a step is about the direction that it can take. There are two possibilities, a forward step, which is the most natural to a human perform, or a backward step.

During this research, by analysing the datasets collected from all the walks, it was identified that step direction can be characterized by combining the data obtained from two sensors placed in different $\mathrm{BSU}$, the foot accelerometer and the waist gyroscope. In the case of the accelerometer, the one placed in the pedestrian's foot gives more accurate results than the one on the waist. However, in the case of the gyroscope, the best results can be achieved with the one placed on the waist, since it give us the pelvic rotation, which combined with the accelerometer data is important to determine the direction of a step.

To classify the step direction 25 features where extracted from the sensors measurements, where 24 are obtained from the accelerometer data and 1 from the gyroscope data. To extract the features from the accelerometer signal, it was divided into 6 equal parts. For each one of these parts the maximum, minimum and mean values were obtained, then it was calculated the slope. 
The other input is obtained from the gyroscope signal, which represents the motion of the waist. If the pelvic as a positive rotation, then the value 1 is assigned to the input, if it is a negative rotation the value 0 is assigned to the input. For example, for a forward left step the rotation will be positive and for a backward left step the rotation will be negative, for the right foot it is the opposite.

To train the learning algorithms the following number of samples was used: 450 samples (190 forward and 260 backward) for training, 100 samples for validation (40 forward and 60 backward) and 100 samples (50 forward and 50 backward) for testing. A 10-fold crossvalidation using these datasets was also performed.

\section{A. SVM}

The design of the implemented SVM approach can be seen in Figure 7. It receives as input the 25 features previously presented. This input is passed to the Hidden Nodes that estimate the best separating hyperplane between the two classes, which maximizes the margin between the two classes. This division is performed using a "linear" kernel. Then, the Output Layer returns the final result about the step direction. A degree of confidence for the two possible results is given by this algorithm.

During the cross-validation the algorithm achieved an accuracy of $100 \%$ for both classes, forward or backward.

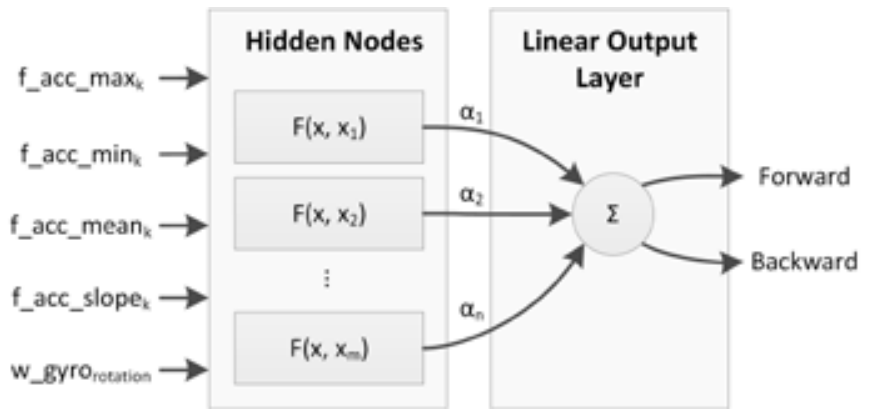

Figure 7 - SVM architecture for step direction characterization

\section{B. Neural Network}

The design of the implemented neural network can be seen in Figure 8 . The neural network receives as input (j) the 25 features previously presented. This input is passed to the Hidden Layer, which is composed by 10 neurons. Then, the Output Layer returns the final result about the step direction.

The learning rate was defined as 0.01 and the number of iterations was defined as 31 .

The mean squared error of the best validation performance is $2.08 \times$ $10^{-8}$ with a gradient of $9.58 \times 10^{-7}$ at epoch 35 . The error is very low when training the network, but even lower when validating and testing the established neural network. Also, more than $90 \%$ of the results are very close to zero error. The highest error for an instance, during the network training, was of 0.15 .

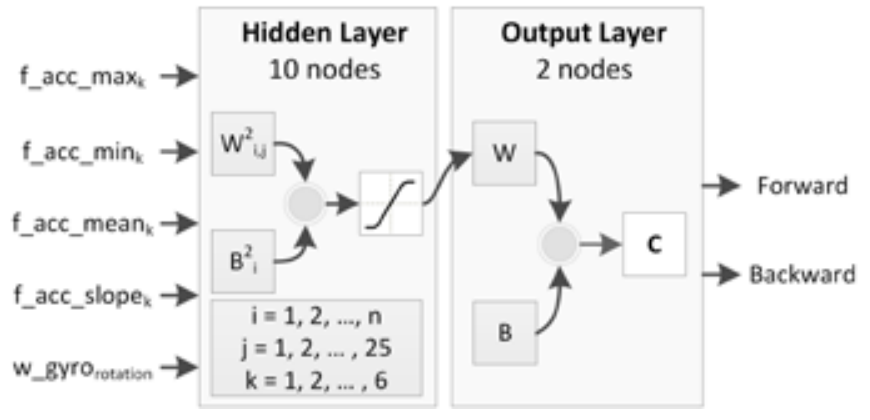

Figure 8 - Neural Network architecture for step direction characterization

\section{Experimental Results}

The step direction characterization algorithms were evaluated using a dataset of 240 steps performed by two pedestrians (120 steps for each pedestrian).

The test scenario is the same as in the previous characterization, where it was walked in forward and in backward movements. Two runs in this scenario, for each pedestrian, were performed.

The results obtained for this scenario can be seen in Table 2. From the obtained results, it can be concluded that, to perform this characterization, the data obtained from the foot BSU sensors are better, than the data obtained from the waist BSU sensors. This mainly happens because when the user is moving the foot is a more stable platform than the waist. A lot of unwanted accelerations are sensed by the waist, which leads to a poor characterization of the step direction, but there are some features that can be retrieved to help other sources to properly characterize the step.

Regarding the step direction characterization the backward one is the most difficult to classify. Mainly because for a human a forward step is a more natural movement to perform than a backward one.

The step direction is a simple characterization to be performed to a step, so the results were the expected. The learning algorithms proved to have an accuracy of $100 \%$.

For this characterization it was not detected any difference in behaviour between the two learning algorithms.

TABLE 2 - ACCURACY RESULTS FOR STEP DIRECTION CHARACTERIZATION

\begin{tabular}{ccccc}
\hline \multirow{2}{*}{ Method } & \multicolumn{2}{c}{ Ascending } & \multicolumn{2}{c}{ Descending } \\
\cline { 2 - 5 } & Waist & Foot & Waist & Foot \\
& BSU & BSU & BSU & BSU \\
\hline SVM & $98.6 \%$ & $100 \%$ & $96.7 \%$ & $100 \%$ \\
\hline N.N. & $99.4 \%$ & $100 \%$ & $95.5 \%$ & $100 \%$ \\
\hline SVM Fusion & \multicolumn{2}{c}{$100 \%$} & \multicolumn{2}{c}{$100 \%$} \\
\hline N.N. Fusion & \multicolumn{2}{c}{$100 \%$} & \multicolumn{2}{c}{$100 \%$} \\
\hline
\end{tabular}

\section{Step Length}

The third, and final, characterization performed to a step is about the length class. There are three possibilities, a short, a normal or a long step. These intervals must be defined from a set of exercises for a pedestrian in specific. Based on the collected data, and on the average of the collected steps, it was considered that short steps are the ones with a maximum distance of $30 \mathrm{~cm}$, the normal steps size ranges between $30 \mathrm{~cm}$ and $45 \mathrm{~cm}$, and the long steps have a size longer than $45 \mathrm{~cm}$.

The $\mathrm{x}$-axis of the foot accelerometer measures the acceleration that is sensed in the horizontal movement of the foot. The quantification of this acceleration is important, because it is correlated with the performed displacement. As the duration and the peak of the acceleration is higher, the longer is the step.

The force sensor data gives reliable information about the amount of time that the foot is not in contact with the ground and, about the force intensity that is made when touching the ground, as well as, when lifting the foot from the ground. The amount of time that the foot is in the air, can be correlated with the acceleration. A higher acceleration value combined with a longer duration of the foot in the air, indicates that a longer step was made.

The $\mathrm{x}$-axis of the gyroscope data gives reliable information about 
the rotation that was performed by the pelvic, where a higher rotation corresponds to a longer step. However, in corners this rotation can be higher for the same type of step. Thus, the combination of this data with the data given by the foot BSU sensors is important to achieve more accurate results. Many errors can occur by using the gyroscope data by itself.

The implemented learning algorithms have as input the foot force sensor and accelerometer data, and the waist gyroscope data. A total of 29 features are fed into the learning algorithms to classify the step length, where 24 are retrieved from the foot accelerometer, 3 from the foot force sensor and 2 from the waist gyroscope.

The foot accelerometer signal was divided into 6 equal parts, as shown in the previous implemented neural networks. This gives a total of 24 inputs that are fed into the learning algorithm.

The next 3 features are obtained from the force sensor signal. The first one is the number of measurements that exist until the maximum force value occur (foot touches the ground). A stronger impact means that the step was longer. The other feature is the force applied when the foot lifts up from the ground. This gives information about the impulse that was performed in the leg in order to perform some horizontal movement. Typically, when the impulse is higher the step is longer. The last feature retrieved from the force sensor is the number of measurements with value of zero, which corresponds to the amount of time that the foot is in the air.

From the gyroscope signal two features are extracted. The first one is the amplitude of the signal, which is correlated to the size of a step. Typically, a higher rotation means that the step is longer. The second feature is the length of the signal, which correlated with the amplitude, gives important information about the size of the step.

From the several tests performed, these features were the ones that had the best results in classifying the possible length of a step.

The learning algorithms were trained with a total of 855 samples, 435 samples of a short step, 225 samples of a normal step and 195 samples of a long step. To validate the algorithms a total of 171 samples were used ( 87 short, 45 normal and 39 long). To test the algorithms a total of 114 samples were used (58 short, 30 normal and 26 long). As in the other characterizations a 10 -fold cross-validation using these datasets was also applied.

\section{A. SVM}

The design of the implemented SVM approach can be seen in Figure 9. This approach receives as input the 29 features previously presented.

In this characterization three SVM models (SVM Model 1, SVM Model 2 and SVM Model 3) were created. After some testing, it was identified that the best results were achieved with the following configuration for each model:

- SVM Model 1 was configured to classify the short steps using a "rbf" (radial basis function or Gaussian) kernel configured with an automatic scale;

- SVM Model 2 was configured to classify the normal steps with a "polynomial" kernel, configured as a $2^{\text {nd }}$ order polynomial;

- SVM Model 3 was configured to classify the long steps using a "linear" kernel.

The models were trained with the same data, but with different class labels vectors. In this case there are three vectors. The first vector, which is used by the SVM Model 1, indicates that the short steps belong to the positive class and the others to the negative. The second vector, which is used by the SVM Model 2, indicates that the normal steps are the positive entries and the others the negatives. The third vector, which is used by the SVM Model 3, indicates that the long steps are the positive classifications and the others the negatives.
The score of the new observations are then estimated using each classifier. This will create a vector with three scores, one per each classifier. The index of the element with the highest score is the index of the class to which the new observation most likely belongs. For example, if the first index has the highest value, then the step is characterized as short. Thus, each new observation is associated with the classifier that gives to it the maximum score.

After the learning phase, a 10-fold cross validation to the model was performed. The SVM Model 1 had no error, the SVM Model 2 presented an error of $7 \%$ and the SVM Model 3 presented an error of $0.8 \%$.

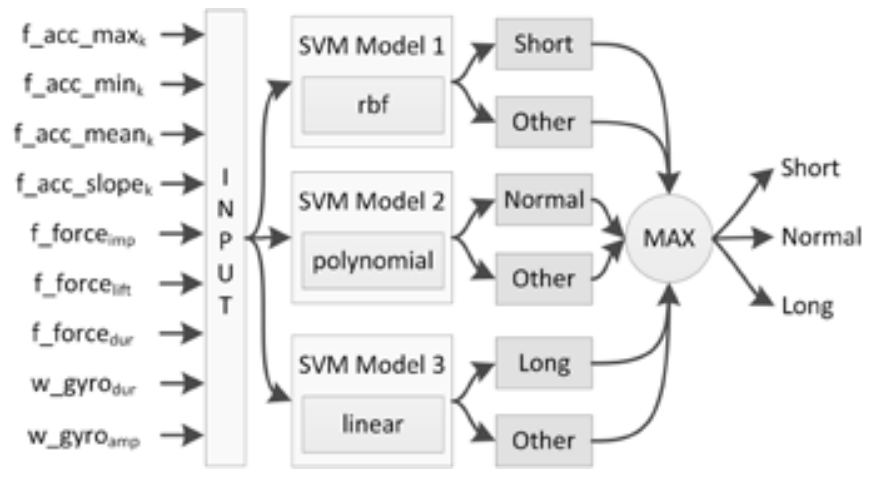

Figure 9 - SVM architecture for step length characterization

\section{B. Neural Network}

The implemented neural network to classify the step length is shown in Figure 10. The neural network receives as input (j) the 29 features previously presented. This input is passed to the Hidden Layer, which is composed by 60 neurons. Then, the Output Layer returns the final result about the step length.

As in the other characterizations the learning rate was defined as 0.01 , and the number of iterations as 25 .

The mean squared error of the best validation performance was 7.55 $\times 10^{-5}$ with a gradient of $4.59 \times 10^{-4}$ at epoch 25 .

During the training phase more than $95 \%$ of the results were very close to zero error, where the highest error for an instance, during the network training, was of 0.20 .

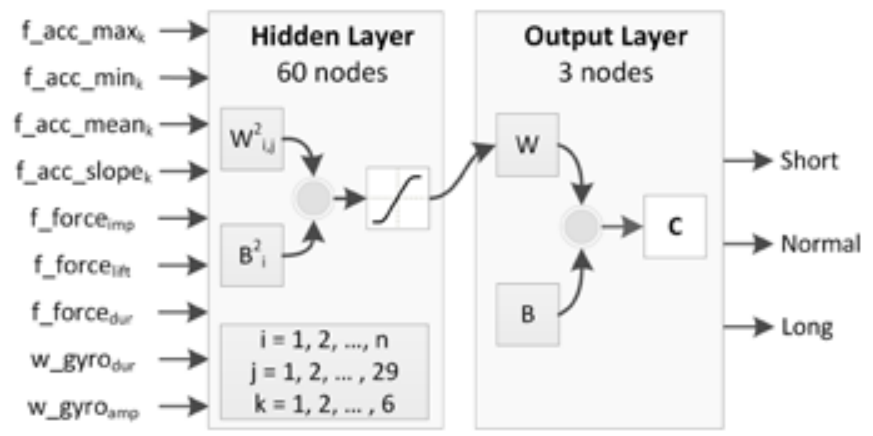

Figure 10 - Neural Network architecture for step length characterization

\section{Evaluation}

The step length characterization algorithms were evaluated using a dataset of 300 steps performed by two pedestrians (150 steps for each pedestrian).

The test scenario is represented in Figure 6 and the obtained results can be seen in Table 3 . 
TABLE 3 - ACCURACY RESULTS FOR STEP LENGTH CHARACTERIZATION

\begin{tabular}{ccccccc}
\hline \multirow{2}{*}{ Method } & \multicolumn{2}{c}{ Short } & \multicolumn{2}{c}{ Normal } & \multicolumn{2}{c}{ Long } \\
\cline { 2 - 7 } & Waist & Foot & Waist & Foot & Waist & Foot \\
& BSU & BSU & BSU & BSU & BSU & BSU \\
\hline SVM & $98.4 \%$ & $96.1 \%$ & $80.3 \%$ & $81.9 \%$ & $92.6 \%$ & $90.7 \%$ \\
\hline N.N. & $98.3 \%$ & $100 \%$ & $86.7 \%$ & $83.3 \%$ & $96.2 \%$ & $100 \%$ \\
\hline SVM Fusion & \multicolumn{2}{c}{$100 \%$} & \multicolumn{2}{c}{$86.0 \%$} & $98.1 \%$ \\
\hline N.N. Fusion & \multicolumn{2}{c}{$100 \%$} & \multicolumn{2}{c}{$90.0 \%$} & \multicolumn{2}{c}{$96.2 \%$} \\
\hline
\end{tabular}

Considering the obtained results, it can be concluded that for a short step both BSU present similar results. For this classification the learning algorithms presented an accuracy of almost $100 \%$.

For a long step, there is not an evident difference between BSUs, since when considering the data from each BSU the Neural Network had the best results. However, when using the data from both BSU, SVM has the best performance.

The normal step is the most difficult to classify. The main reason for this phenomenon is because it sits between the other two classes. For this classification the combination of both BSU gives better results than using the data from each BSU individually. Nonetheless, none of the misclassifications given by the algorithms was to the opposite class, meaning that a short step was never classified as a long step and vice-versa.

Analysing the obtained results, it can be concluded that through the sensors complementarity the step length was categorized with higher accuracy. Also, it can be concluded that the learning of the gait parameters enables a more precise characterization of a step. The Neural Network gave the best results, having a mean accuracy very close to $96 \%$.

From our tests it was identified that a learned dataset 10 times smaller is sufficient to achieve similar results. Making the learning procedure simpler and faster to a pedestrian perform before using this system.

The tests and the evaluation results, have shown that both BSU give similar results on detecting short steps, but in the case of normal steps the foot BSU has a higher accuracy. However, the long steps are better detected by the waist BSU.

Combining the data from both BSU, the weaknesses of one are suppressed by the advantages of the other, thus improving the overall results.

\section{CONCLUSION}

Develop a PINS to be used by pedestrians in their daily life is a huge challenge. Many approaches already have been proposed, but must of them rely on a structured environment that usually is infeasible to implement and the others don't provide the necessary accuracy.

To suppress some of these limitations we propose a PINS based on low-cost sensors and on fusion and learning techniques. The sensors are placed on the foot and on the waist of a pedestrian, and their information is combined to achieve more accurate location estimation results. The data from both IMU was heavily explored in order to provide an acceptable level of performance, since one IMU can complement the other in the different activities that a pedestrian can perform.

The proposed system characterizes the step according to the activity that the pedestrian is performing. This characterization starts by estimating the type of terrain where the step was given. Then it estimates if the step was a forward or a backward one. This is very important to correctly estimate the pedestrian displacement, since they are opposite directions. The third classification is regarding the step length. This characterization fits into one of three categories: short, normal or long. With this classification we limit the displacement estimation according to the bounds of each category.

The inclusion of the step characterization module, through the use of more than one IMU and the neural network algorithm, led to an improvement, compared to the previous results [7], in displacement estimation of $52 \%$. In the same scenario the error has decreased from $7.3 \%$ to $4.8 \%$.

In the future we want to divide the step length characterization into more classes, to verify if it improves the displacement estimation accuracy. Also, we want to implement more step characterization characteristics.

\section{REFERENCES}

[1] J. Elwell, "Inertial navigation for the urban warrior," in Proceedings of SPIE, vol. 3709, 1999, pp. $196-204$.

[2] J. Lucas, N. Luz, M. Moreno, R. Anacleto, A. Almeida, and C. Martins, "A hybrid recommendation approach for a tourism system," Expert Systems with Applications, vol. 40, no. 9, pp. 3532 - 3550, Jul. 2013.

[3] J. Ramos, R. Anacleto, A. Costa, P. Novais, L. Figueiredo, and A. Almeida, "Orientation system for people with cognitive disabilities," in Ambient Intelligence - Software and Applications, ser. Advances in Intelligent and Soft Computing. Springer Berlin Heidelberg, Jan. 2012, no. 153, pp. 43 -50 .

[4] R. Anacleto, N. Luz, A. Almeida, L. Figueiredo, and P. Novais, "Shopping center tracking and recommendation systems," in Soft Computing Models in Industrial and Environmental Applications, 6th International Conference SOCO 2011, ser. Advances in Intelligent and Soft Computing. Springer Berlin Heidelberg, 2011, no. 87, pp. $299-308$.

[5] J. Hightower and G. Borriello, "Location systems for ubiquitous computing," IEEE Computer, vol. 34, no. 8, pp. 57 - 66, 2001.

[6] R. Anacleto, L. Figueiredo, A. Almeida, and P. Novais, "Person localization using sensor information fusion," in Ambient Intelligence - Software and Applications, ser. Advances in Intelligent Systems and Computing. Springer International Publishing, Jan. 2014, no. 291, pp. 53-61.

[7] R. Anacleto, L. Figueiredo, A. Almeida, and P. Novais, "Localization system for pedestrians based on sensor and information fusion," in 17th International Conference on Information Fusion (FUSION), July 2014, pp. $1-8$.

[8] M. Liggins, D. Hall, and J. Llinas, Handbook of Multisensor Data Fusion: Theory and Practice, Second Edition, 2nd ed. CRC Press, 1997.

[9] K. Faceli, A. d. Carvalho, and S. O. Rezende, "Combining Intelligent Techniques for Sensor Fusion," Applied Intelligence, vol. 20, no. 3, pp. 199-213, May 2004.

[10] S. Thrun, M. Montemerlo, H. Dahlkamp, D. Stavens, A. Aron, J. Diebel, P. Fong, J. Gale, M. Halpenny, G. Hoffmann, K. Lau, C. Oakley, M. Palatucci, V. Pratt, P. Stang, S. Strohband, C. Dupont, L.-E. Jendrossek, C. Koelen, C. Markey, C. Rummel, J. van Niekerk, E. Jensen, P. Alessandrini, G. Bradski, B. Davies, S. Ettinger, A. Kaehler, A. Nefian, and P. Mahoney, "Stanley: The robot that won the DARPA Grand Challenge," Journal of Field Robotics, vol. 23, no. 9, pp. 661-692, Sep. 2006.

[11] F. Caron, E. Duflos, D. Pomorski, and P. Vanheeghe, "GPS/IMU Data Fusion Using Multisensor Kalman Filtering: Introduction of Contextual Aspects," Information Fusion, vol. 7, no. 2, pp. 221-230, Jun. 2006.

[12] A. Noureldin, T. B. Karamat, M. D. Eberts, and A. El-Shafie, "Performance Enhancement of MEMS-Based INS/GPS Integration for Low-Cost Navigation Applications," IEEE Transactions on Vehicular Technology, vol. 58, no. 3, pp. 1077-1096, Mar. 2009.

[13] A. Noureldin, A. El-Shafie, and M. Bayoumi, "GPS/INS integration utilizing dynamic neural networks for vehicular navigation," Information Fusion, vol. 12, no. 1, pp. 48-57, Jan. 2011.

[14] D. Bhatt, P. Aggarwal, V. Devabhaktuni, and P. Bhattacharya, "A novel hybrid fusion algorithm to bridge the period of GPS outages using lowcost INS," Expert Systems with Applications, vol. 41, no. 5, pp. 21662173, Apr. 2014. 
[15] STMicroelectronics, "L3g4200d: Three axis digital output gyroscope," 2014. Available: http://www.st.com/st-web-ui/static/active/en/resource/ technical/document/datasheet/CD00265057.pdf.

[16] A. Devices, “Adx1345 digital accelerometer," 2014. [Online]. Available: http://www.analog.com/static/imported-files/data sheets/ADXL345.pdf.

[17] Honeywell, "3-axis digital compass ic hmc58831," 2014. Available: http:/ www51.honeywell.com/aero/common/documents/myaerospacecatalogdocuments/Defense Brochures-documents/HMC5883L 3-Axis Digital Compass IC.pdf.

[18] Tekscan, "Flexiforce sensors for force measurement," 2014. [Online]. Available: http://www.tekscan.com/flexible-force-sensors.

[19] J. Saunders, V. Inman, and H. Eberhart, "The major determinants in normal and pathological gait," The Journal of Bone \& Joint Surgery, vol. 35 , no. 3, pp. $543-558,1953$.

[20] C. Vaughan, B. Davis, and J. O'connor, Dynamics of human gait. Human Kinetics Publishers Champaign, Illinois, 1992.

[21] C. Mateos, C. Ruiz, R. Crespo and A. Sanz, "Relative Radiometric Normalization of Multitemporal Images", International Journal of Artificial Intelligence and Interactive Multimedia, Vol 1, no. 3, pp. 54-59, 2010

[22] S. Rios-Aguilar, "Intelligent Position Aware Mobile Services for Seamless and Non-Intrusive Clocking-in", International Journal of Interactive Multimedia and Artificial Intelligence, Vol. 2, no. 5, pp. 48-50, 2014

[23] J. Espada, V. García-Díaz, R. Crespo, B. G-Bustelo, J. Lovelle, "Improving the GPS Location Quality Using a Multi-agent Architecture Based on Social Collaboration", Pratical Applications of Intelligent Systems, Advances in Intelligent Systems and Computing, Springer Berlin Heidelberg, Vol. 279, pp. 371-379, 2014

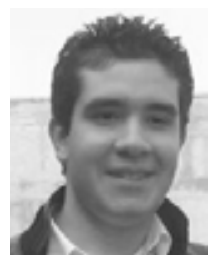

Ricardo Anacleto. Since 2011 it is a PhD student in the Doctoral Program MAP-i in the areas of Mobile Computing and Localization Systems. It teaches in ESTGF - School of Technology and Management Felgueiras, teaching in the area of Computer Science (Mobile and Ubiquitous Computing). Currently, he is also a member of the ALGORITMI research group at University of Minho.

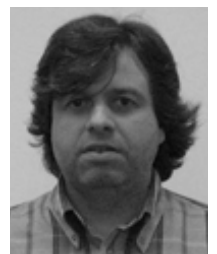

Lino Figueiredo received the Ph.D degree in Electrical and Computer Engineering from the Faculty of Engineering, University of Porto, Portugal, in 2005. He is a Professor with the Electrical Engineering Department, Institute of Engineering of Porto and member of the Knowledge Engineering and Decision Support (GECAD) research group. His current research interests include ambient intelligence, Wireless intelligent sensors, Simulation and modeling of traffic systems and intelligent transportation systems.

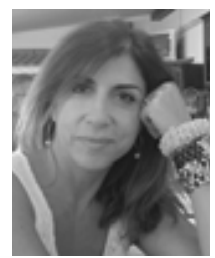

Ana Almeida obtained a $\mathrm{PhD}$ in Production end Systems Engineering in 2003, from University of Minho. Her research interests include AI applications, Intelligent Systems, Mobile Systems and Affective Computing. She is a member of the Knowledge Engineering and Decision Support (GECAD) research group. She has participated in more than fifteen projects and integrated the organizing/ program/scientific committee of several scientific conferences.

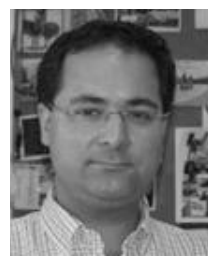

Paulo Novais is an Associate Professor with Habilitation of Computer Science at the Department of Informatics, in the School of Engineering of the University of Minho (Portugal) and a researcher at the ALGORITMI Centre in which he is the coordinator of the research group Intelligent Systems Lab, and the coordinator of the research line in "Ambient intelligence for well-being and Health Applications".

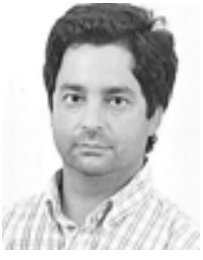

António Meireles is a $\mathrm{PhD}$ student in Electrical Engineering and Signal Processing at University of Aveiro and researcher at research group GECAD. He has a Master degree in Electronics Engineering from ISEP, a post-graduation in Biomedical Engineering from University of Porto and management from Porto Business School. During his career he was project leader in different research and development projects and semiconductor development. His main interests are electronics and digital signal processing applied to biomedical devices. 